\title{
Race, sense of control over life, and short-term risk of mortality among older adults in the United States
}

Shervin Assari

Department of Psychiatry and Center for Research on Ethnicity, Culture, and Health (CRECH), University of Michigan, Ann Arbor, USA

Submitted: 5 February 2016

Accepted: 17 April 2016

Arch Med Sci 2017; 13, 5: 1233-1240

DOI: $10.5114 /$ aoms.2016.59740

Copyright $\odot 2016$ Termedia \& Banach

\section{Abstract}

Introduction: Sense of control over life has been shown to have protective health effects in studies that have mostly enrolled White middle class individuals. It is unknown, however, whether populations differ in the protective health gain associated with sense of control over life. This study compared a nationally representative sample of Black and White older adults for protective effects of sense of control over life on short-term risk of all-cause mortality in the United States.

Material and methods: This longitudinal prospective study followed 1,493 White $(n=759)$ and Black $(n=734)$ older adults (age 66 or more) from 2001 to 2004. Race, demographics, socio-economics, sense of control over life, health behaviors, and self-rated health were measured at baseline in 2001 . Outcome was all-cause mortality occurring between 2001 and 2004. Logistic regression models were used for data analysis.

Results: In the pooled sample, sense of control over life was protective against 3-year mortality risk above and beyond demographics, socio-economics, health behaviors, and self-rated health. We found a race by sense of control over life interaction, suggesting a stronger protective effect of control over life on mortality risk for Whites compared to Blacks. In race-specific models, sense of control over life at baseline was predictive of mortality among Whites but not Blacks.

Conclusions: In the United States, Black older adults do not gain a survival benefit associated with high levels of sense of control over life, as do their White counterparts. It is not clear why sense of control over life translates into survival for Whites but not for Blacks.

Key words: health disparities, ethnic groups, African Americans, Blacks, mortality, control over life.

\section{Introduction}

Similar to other psychological constructs such as locus of control, self-efficacy, mastery, self-directedness, personal autonomy, instrumentalism, and helplessness, which have been used interchangeably [1], sense of control over life also predicts better health status [2]. Individuals with a high sense of control over life report higher well-being [3] and lower stress, anxiety, and depression [4]. A high level of control is also associated with better physical health outcomes such as physical functioning [5] as well as lower risk of cardiovascular disease [6]. A high level of control over life also predicts lower risk of mortality [7].

Sense of control over life - also known as perceived control, control beliefs, or sense of control - refers to subjective expectations regard-

\author{
Corresponding author: \\ Shervin Assari MD, MPH \\ Department of Psychiatry \\ and Center for Research on \\ Ethnicity, Culture, \\ and Health (CRECH) \\ University of Michigan \\ 2402 Pittsfield Blvd \\ 48104 Ann Arbor, USA \\ Phone: 1-734-363-2678 \\ E-mail: assari@umich.edu
}


ing one's ability to exert an influence over life circumstances and outcomes in the surrounding environment [2]. Sense of control over life is a cognitive style which reflects whether life outcomes are subjectively ascribed to the self vs. something external to a person. While shaped by age, gender [8], life events [9], and sociological and contextual factors such as race, education, and marriage [1], sense of control is frequently viewed as a stable personality trait [10]. In this view, individuals are located on a continuum, from having the generalized belief that one can and does master, control, and shape one's own life, to the opposite end of the continuum, which is perceived powerlessness, accompanied by the belief that one's life is shaped by external forces, such as luck, chance, fate, or powerful others [7, 11].

In 2014, Turiano et al. studied population variation in the protective effects of sense of control over life on mortality risk. The authors found that education level alters how sense of control over life predicts risk of all-cause mortality. Control beliefs were a predictor of mortality risk only among individuals with low but not high education. The authors also showed that such an interaction is not due to confounders such as health behaviors, mood, or health status [7]. Although in the U.S. race is a strong proxy of social class [12], it is still unknown whether race moderates the protective effect of control beliefs. In other terms, it is unknown whether Blacks and Whites differ in the health gain associated with such control beliefs.

In 2016, Assari used follow-up data of 3,361 Black $(n=1,156)$ or White $(n=2,205)$ adults for up to 25 years from 1986 to 2011 . The author measured self-efficacy in 1986 and used Cox proportional hazards models to test whether the effect of self-efficacy beliefs on risk of mortality depends on race. The author found a stronger protective effect of self-efficacy on mortality for Whites compared to Blacks. In race-specific models, self-efficacy protected Whites but not Blacks against mortality. The author concluded that in the United States, longterm health gains associated with high self-efficacy are not universal but race-specific [13].

In response to the very limited knowledge on group differences in the protective effect of control beliefs on mortality, the current study compared Blacks and Whites for the effects of sense of control over life on short-term risk of mortality in the United States. To provide generalizable results, we used nationally representative data.

\section{Material and methods}

\section{Design and setting}

This was a longitudinal panel study with three years of follow-up. Data came from wave 1 and wave 2 of the Religion, Aging, and Health Survey, a household survey from 2001 until 2004 [14].

\section{Ethics}

The project received Institutional Review Board (IRB) approval from the University of Michigan. All participants provided consent.

\section{Participants}

The study only included White and Black older adults. All participants were non-institutionalized English speaking people of age more than 65 years. Geographically, the study population was restricted to individuals residing in the coterminous United States (i.e., residents of Alaska and Hawaii were not sampled). The study population was limited to Christians or those who were never associated with any faith. Older Blacks were oversampled in the survey [14].

\section{Sampling frame}

The study used a random sampling strategy. The sampling frame for this study consisted of all eligible persons contained in the Medicare Beneficiary list maintained by the Centers for Medicare and Medicaid Services (CMS). A five-step process was used to draw a sample from the CMS file (54).

\section{Interviews}

Data collection was performed by Louis Harris and Associates (now Harris Interactive, New York). Interviewing began in March 2001 and concluded in August 2001 [14].

\section{Measures}

Age, race, family income, number of chronic medical conditions (13 chronic medical conditions), perceived financial difficulty, self-rated health, perceived control over life, and death anxiety were measured.

Perceived control over life. The following four items were used to measure control over life. 1) I have a lot of influence over most things that happen in my life. 2) I can do just about anything I really set my mind to. 3) When I make plans, I'm almost certain to make them work. 4) When I encounter problems, I don't give up until I solve them. Responses ranged from 4 (Strongly Agree) to 1 (Strongly Disagree). A higher score reflected higher control over life. (Cronbach's $\alpha=0.743$ ) [15].

Self-rated health (poor). Individuals were asked three questions. 1) How would you rate your overall health at the present time? Would you say your health is excellent, good, fair, or poor? 2) Would you say your health is better, about the same, or worse than most people your age? 3) Do you think 
your health is better, about the same, or worse than it was a year ago? Responses to the first item included: 1) Excellent, 2) Good, 3) Fair, 4) Poor. Responses to the second and third items included 1) Better, 2) About the same, and 3) Worse (Cronbach's $\alpha=0.678$ ).

Mortality. Information on occurrence of death from 2001 to 2004 was obtained through the informants, death certificates, and national death index. Mortality during the follow-up was treated as a dichotomous variable, independent of time and cause of death. Overall, 208 deceased Black or White participants were detected. From this number, 112 were Black and 96 were White.

\section{Statistical analysis}

We used SPSS 20.0 for data analysis. We fitted logistic regressions in the pooled sample, and specific to race, with sense of control as the independent variable, mortality as the outcome, and demographics, socio-economics, health behaviors, and self-rated health as covariates, and race as the focal moderator. Odds ratios with $95 \%$ confidence intervals $(\mathrm{Cl})$ were reported.

\section{Results}

The study followed 1,493 Black $(n=734)$ and White ( $n=759$ ) older adults (age 65 or more) for 3 years. Overall, 208 deceased participants were detected. From this number, 112 (54\%) individuals were Blacks and 96 (56\%) individuals were Whites.

Table I presents descriptive statistics overall and also based on race. While age was not significantly different between Blacks and Whites, Blacks were more often female, had lower education, were less frequently married, more frequent-

Table I. Descriptive statistics for the analytic sample, stratified by race and overall

\begin{tabular}{|c|c|c|c|}
\hline Parameter & All & Whites & Blacks \\
\hline Age, mean \pm SD & $75.14 \pm 6.66$ & $75.37 \pm 6.82$ & $74.91 \pm 6.49$ \\
\hline Control over life, mean $\pm \mathrm{SD}^{*}$ & $1.98 \pm 0.51$ & $2.02 \pm 0.47$ & $1.93 \pm 0.54$ \\
\hline \multicolumn{4}{|l|}{ Gender, $n(\%):^{*}$} \\
\hline Male & $570(38.2)$ & $314(41.4)$ & $256(34.9)$ \\
\hline Female & $923(61.8)$ & $445(58.6)$ & $478(65.1)$ \\
\hline \multicolumn{4}{|l|}{ Education (high school diploma): } \\
\hline Yes & $872(59.0)$ & $552(73.4)$ & $320(44.0)$ \\
\hline No & $607(41.0)$ & $200(26.6)$ & $407(56.0)$ \\
\hline \multicolumn{4}{|l|}{ Marital status (married), $n$ (\%):* } \\
\hline No & $773(52.2)$ & $306(40.5)$ & $467(64.3)$ \\
\hline Yes & $708(47.8)$ & $449(59.5)$ & $259(35.7)$ \\
\hline \multicolumn{4}{|l|}{ Current smoking, $n(\%):^{*}$} \\
\hline Yes & $155(10.4)$ & $60(7.9)$ & $95(13.0)$ \\
\hline No & $1336(89.6)$ & $698(92.1)$ & $638(87.0)$ \\
\hline \multicolumn{4}{|l|}{ Drinking (lifetime), $n(\%):^{*}$} \\
\hline Yes & $466(31.3)$ & $307(40.5)$ & $159(21.7)$ \\
\hline No & $1025(68.7)$ & $451(59.5)$ & $574(78.3)$ \\
\hline \multicolumn{4}{|l|}{ Self-rated health (poor), $n$ (\%):* } \\
\hline No & $1316(88.4)$ & $694(91.8)$ & $622(84.9)$ \\
\hline Yes & 173 (11.6) & $62(8.2)$ & $111(15.1)$ \\
\hline \multicolumn{4}{|l|}{ Mortality, $n$ (\%): } \\
\hline Survived & $1285(86.1)$ & $663(87.4)$ & $622(84.7)$ \\
\hline Deceased & $208(13.9)$ & 96 (12.6) & $112(15.3)$ \\
\hline
\end{tabular}

${ }^{\star} P<0.05$ 


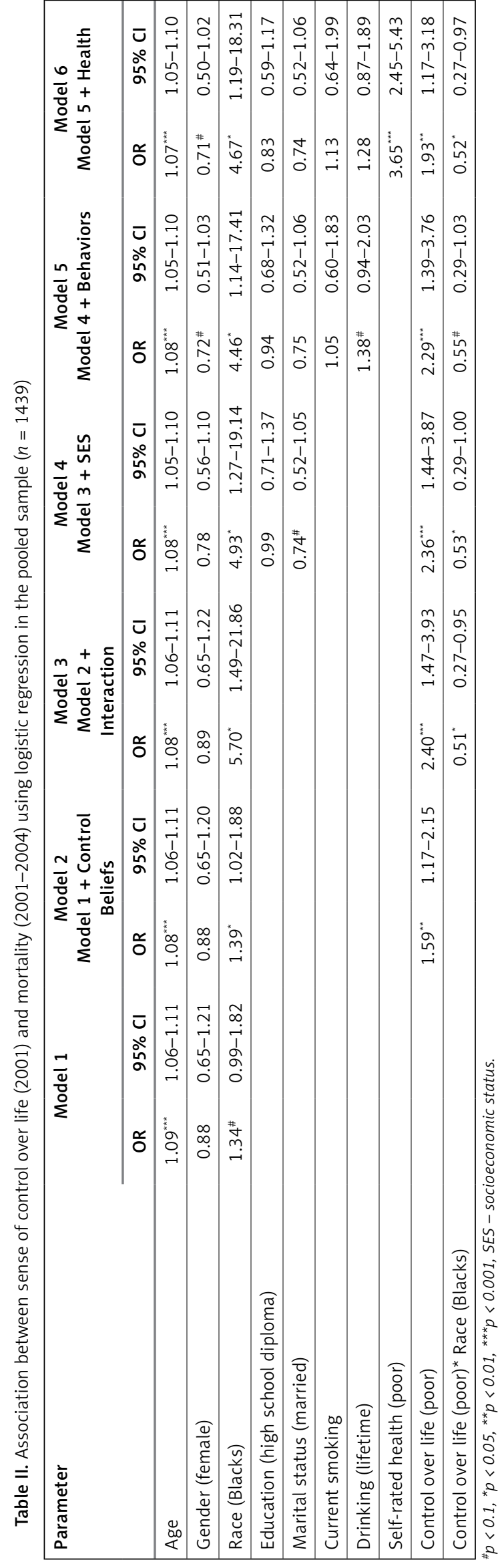

ly smoked, less frequently drank, and reported worse self-rated health. Sense of control over life was also lower among Blacks compared to Whites.

Table II shows the results of six logistic regressions in the pooled sample. While sense of control over life was a predictor of mortality (Model 2), we also found significant interaction effects between race and sense of control over life on mortality, suggesting larger effect for Whites compared to Blacks (Model 3). This interaction remained significant in models that controlled for covariates (Models 4 to 6).

Table III presents race-specific models to estimate the associations between sense of control over life and subsequent risk of mortality among Blacks and Whites. Model 1 only included age and gender, Model 2 added control beliefs, Model 3 added SES factors, Model 4 also added health behaviors (smoking and drinking), and Model 5 also controlled for health status. These models suggest that sense of control over life at baseline was predictive of mortality among Whites but not Blacks. The significant association between control over life and risk of mortality among Whites did not change across models, suggesting that the association was not due to SES, behaviors, or health. Among Blacks, in all five models, control over life failed to predict risk of mortality.

\section{Discussion}

Based on our findings, race alters how sense of control over life translates into survival benefit, with a weaker protective effect of sense of control over life on short-term mortality risk for Blacks compared to Whites. In race-specific models, self-efficacy was only protective against mortality among Whites but not Blacks.

Our finding is in line with a recent study by Assari showing a stronger protective role of self-efficacy on risk of mortality over 25 years for Whites compared to Blacks. In race-specific models, self-efficacy at baseline was predictive of mortality among Whites but not Blacks. The author concluded that in the United States, race modifies long-term health gains associated with high self-efficacy [13]. The current study suggests that, similar to self-efficacy, sense of control over life better predicts risk of mortality for Whites compared to Blacks. Our study also suggests that Black-White differences in gain associated with such control beliefs and psychological and cognitive styles are not seen in either long-term [13] or short-term outcomes.

The protective role of having a sense of control over life against risk of mortality has already been shown $[6,7]$. Such an effect may be due to protective effects of control beliefs on developing psychological distress, as well as metabolic [16] 


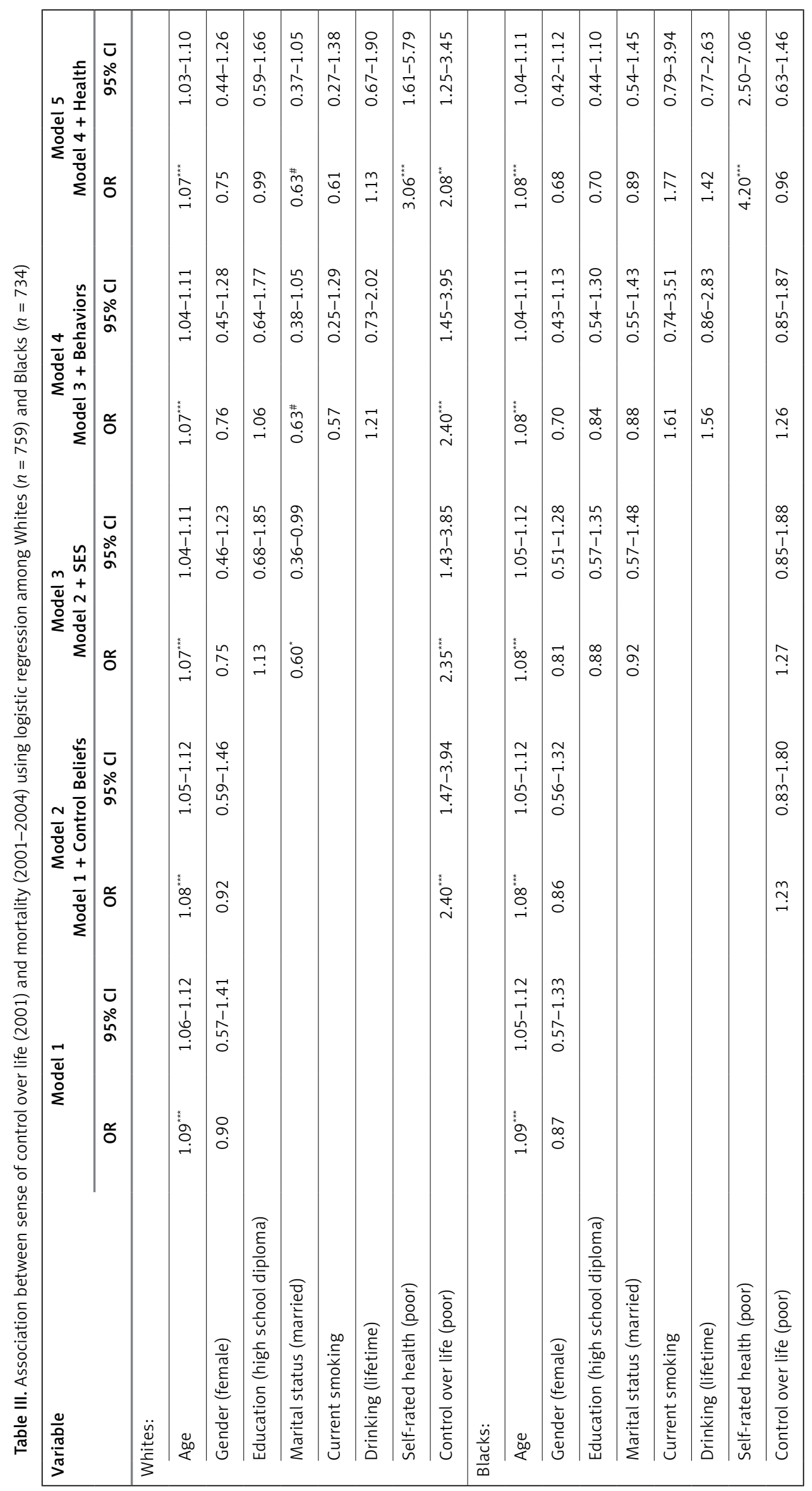


and cardiovascular [17] disease. Such beliefs also reflect lower symptoms [18], better wellbeing [18], and higher physical functioning $[18,19]$. Low control beliefs reflect feeling of powerless and low efficacy in dealing with stress [20] as well as high levels of exposure to stress [21]. Among individuals of low socioeconomic status, those with higher control beliefs have better self-rated health, fewer acute health symptoms, and better physical functioning compared to those with lower levels of control beliefs [22]. The protective effect of self-efficacy is shown to hold above and beyond confounders such as SES and health [23, 24].

Several mechanisms have been proposed to explain the protective effects of control beliefs on health. Health behaviors and mental health are major mediators of the effects of control beliefs on health $[4,7]$. Control beliefs may mitigate the negative health effects of stress or structural and material limitations that often accompany low socioeconomic status [22]. According to the Lazarus and Folkman theory of stress and coping, at least some of the negative health effects of stress are because of reduced individuals' perception of control over life [25]. While some of the deleterious effects of stress on health are direct, some of the negative effects may be due deterioration of positive appraisals such as mastery, self-efficacy, and sense of control over life [25].

Our findings on racial differences in protective effects of sense of control may be due to different meanings of control beliefs across population groups. Control beliefs may reflect different aspects of life of Blacks and Whites [13, 26, 27]. The differential roles of other psychosocial and subjective measures such as depressive symptoms and self-rated health for Blacks and Whites are already known [28-33]. Thus group differences in the predictive role of psychosocial outcomes are rules rather than exceptions and are found regardless of population, setting, type of predictor, and outcome [29-33].

The low sense of control over life among Whites and Blacks may reflect different aspects of life. Given the social and economic conditions that Blacks face on a daily basis, low control beliefs may reflect a healthy sensitivity to the real world (realistic system-blame). For Blacks, low sense of control over time may be an attribute of their racial group, rather than their individual characteristic. Among Blacks, low sense of control may be secondary to realization of the existing social and economic inequalities in the U.S. Thus, such control beliefs may not similarly reflect passive beliefs, external locus of control or fatalism among Blacks and Whites [34].

According to our findings, high sense of control does not result in similar survival gains for Black and White older adults. Under certain envi- ronmental circumstances, high level of control beliefs has predicted poor health outcomes $[35,36]$. Thus, control beliefs may even harm the individual, particularly when high expectations for control coexist with constrained opportunities [36, 37]. In uncontrollable or difficult to control situations, endorsement of high control beliefs can lead to high cardiovascular reactivity $[38,39]$, which is associated with higher risk of metabolic and cardiovascular conditions, via neuroendocrine and immune mechanisms [38-40]. While societal structural conditions prevent Blacks who endorse high control beliefs from actualization of their control beliefs, Blacks with high control beliefs may be at higher risk of cardiovascular disease due to experiencing high levels of physiological reactivity [35] For Blacks a combination of constrained opportunities and high control beliefs may do more harm than good. This phenomenon may also be related to the role of John Henryism for Blacks, which results in effortful coping, and is a risk factor for several adverse health outcomes [41].

Our study has a number of limitations. It is unclear how such control beliefs reflect coping styles, coping resources and life environment across diverse populations. Validity of control over life may differ based on race and ethnicity. In addition, the study did not control for baseline medical conditions and stressful life events, or access to and use of health care. Finally, due to the limited sample size and short follow-up period, the number of deaths was low. However, a major strength of this study was the use of a nationally representative sample.

In conclusion, Black and White older adults differ in benefits of high levels of control beliefs in preventing short-term all-cause mortality. Control beliefs may reflect different aspects of life across minority and oppressed populations. For American Blacks, a high level of self-efficacy may reflect a low level of awareness of blocked opportunities and racism, which may not be a healthy realization. Such psychosocial constructs may have race-specific - rather than universal - health effects.

\section{Acknowledgments}

Funding: The Religion, Aging, and Health Survey was supported by the National Institute on Aging (PI: Neal Krause; R01 AG014749), and per the NIH Public Access Policy requires that peer-reviewed research publications generated with $\mathrm{NIH}$ support are made available to the public through PubMed Central. NIH is not responsible for the data collection or analyses represented in this article. Data were accessed through the Interuniversity Consortium for Political and Social Research (ICPSR), Institute of Social Research, University of Michigan. 
Shervin Assari is supported by the Heinz C. Prechter Bipolar Research Fund and the Richard Tam Foundation at the University of Michigan Depression Center.

Ethics: All procedures followed were in accordance with the ethical standards of the responsible committee on human experimentation (institutional and national) and with the Helsinki Declaration of 1975 , as revised in 2000. The University of Michigan Institutional Review Board (IRB) approved the study protocol.

Informed consent: Informed consent was obtained from all participants included in the study.

\section{Conflict of interest}

The author declares no conflict of interest.

\section{References}

1. Ross C, Sastry J. The sense of personal control: socialstructural causes and emotional consequences. In: Handbook of the sociology of mental health. Aneshensel CS, Phelan JC (eds). Kluwer Academic/Plenum, New York 1999; 369-94.

2. Lachman ME, Neupert SD, Agrigoroaei S. The relevance of control beliefs for health and aging. In: Handbook of the psychology of aging. Schaie KW, Willis SL (eds). Elsevier, New York 2011; 175-90.

3. Mirowsky J, Ross CE. Well-being across the life course. In: A handbook for the study of mental health: social contexts, theories, and systems. Horwitz AV, Scheid TL (eds). Cambridge University Press, New York 1999; 328-47.

4. Keeton CP, Perry-Jenkins M, Sayer AG. Sense of control predicts depressive and anxious symptoms across the transition to parenthood. J Fam Psychol 2008; 22: 212-21.

5. Infurna FJ, Gerstorf D, Zarit SH. Examining dynamic links between perceived control and health: longitudinal evidence for differential effects in midlife and old age. Develop Psychol 2011; 47: 9-18.

6. Surtees PG, Wainwright WJ, Luben R, Wareham NJ, Bingham S, Khaw KT. Mastery is associated with cardiovas cular disease mortality in men and women at apparently low risk. Health Psychol 2010; 29: 412-20.

7. Turiano NA, Chapman BP, Agrigoroaei S, Infurna FJ, Lachman M. Perceived control reduces mortality risk at low, not high, education levels. Health Psychol 2014; 33: 883-90.

8. Ross C, Mirowsky J. Age and the gender gap in the sense of personal control. Social Psychology Quarterly 2002; 65: $125-45$

9. Sirignano S, Lachman M. Personality change during the transition to parenthood: the role of perceived infant temperament. Develop Psychol 1985; 21: 558-67.

10. Wolfle LM, List JH. Temporal stability in the effects of college attendance on locus of control, 1972-1992. Structural Equation Modeling 2004; 11: 244-60.

11. Mirowsky J, Ross CE. Eliminating defense and agreement bias from measures of the sense of control: a $2 \times$ 2 index. Social Psychology Quarterly 1991; 54: 127-45.

12. Drake KA, Galanter JM, Burchard EG. Race, ethnicity and social class and the complex etiologies of asthma. Pharmacogenomics 2008; 9: 453-62.

13. Assari S. Ethnic differences in long term predictive role of self-efficacy on risk of mortality in the United States.
Social Psychiatry and Psychiatric Epidemiology 2016 Under review.

14. Krause N. Religion, aging, and health survey, 2001, 2004 [United States]. ICPSR03255-v2. Ann Arbor, MI: Inter-university Consortium for Political and Social Research [distributor], 2006-04-17. http://doi.org/ 10.3886/ICPSR03255.v2.

15. Bradshaw M, Ellison CG, Fang Q, Mueller C. Listening to religious music and mental health in later life. Gerontologist 2015; 55: 961-71.

16. Infurna FJ, Gerstorf D. Perceived control relates to better functional health and lower cardio-metabolic risk: the mediating role of physical activity. Health Psychol 2014; 33: 85-94.

17. Surtees PG, Wainwright WJ, Luben R, Wareham NJ, Bingham S, Khaw KT. Mastery is associated with cardiovascular disease mortality in men and women at apparently low risk. Health Psychology 2010; 29: 412-20.

18. Lachman ME, Weaver SL. The sense of control as a moderator of social class differences in health and well-being. J Personal Soc Psychol 1998; 74: 763-73.

19. Lachman ME, Agrigoroaei S. Promoting functional health in midlife and old age: long-term protective effects of control beliefs, social support, and physical exercise. PLoS One 2010; 5: e13297.

20. Seeman M, Lewis S. Powerlessness, health and mortality: a longitudinal study of older men and mature women. Soc Sci Med 1995; 41: 517-25.

21. Sandler IN, Lakey B. Locus of control as a stress moderator: the role of control perceptions and social support. Am J Comm Psychol 1982; 10: 65-80.

22. Lachman ME, Weaver SL. The sense of control as a moderator of social class differences in health and well-being. J Personal Soc Psychol 1998; 74: 763-73.

23. Penninx BWJH, van Tilburg T, Kriegsman DMW, Deeg DJH, Boeke AJP, van Eijk JT. Effects of social support and personal coping resources on mortality in older age: the Longitudinal Aging Study Amsterdam. Am J Epidemiol 1997; 146: 510-9.

24. Infurna FJ, Ram N, Gerstorf D. Level and change in perceived control predict 19-year mortality: findings from the Americans' changing lives study. Dev Psychol 2013; 49: 1833-47.

25. Lazarus RS, Folkman S. Stress, appraisal, and coping. Springer, New York 1984.

26. Hulbary W. Race, deprivation, and adolescent self-image. Social Science Quarterly 1975; 56: 105-14.

27. Crain RL, Weisman CS. Discrimination, personality, and achievement. Academic Press, New York 1972.

28. Assari S, Burgard SA. Black-White differences in the effect of baseline depressive symptoms on deaths due to renal diseases: 25 year follow up of a nationally representative community sample. J Renal Inj Prev 2015; 4: 127-34.

29. Assari S, Lankarani MM, Burgard S. Black-white difference in long-term predictive power of self-rated health on all-cause mortality in United States. Ann Epidemiol 2016; 26: 106-14

30. Assari S, Moazen-Zadeh E, Lankarani MM, Micol-Foster V. Race, depressive symptoms, and all-cause mortality in the United States. Front Public Health 2016; 4: 40.

31. Assari S, Burgard S, Zivin K. Long-term reciprocal associations between depressive symptoms and number of chronic medical conditions: longitudinal support for Black-White health paradox. J Racial Ethn Health Disparities 2015; 2: 589-97.

32. Lankarani MM, Assari S. Association between number of comorbid medical conditions and depression among 
individuals with diabetes: race and ethnic variations. J Diabetes Metab Disord 2015; 14: 56.

33. Assari S, Lankarani MM. Association between stressful life events and depression: intersection of race and gender. J Racial Ethnic Health Dispar 2015; 2: 1-8.

34. Hughes M, Demo DH. Self-perceptions of Black Americans: self-esteem and personal efficacy. Am J Sociol 1989; 1: 132-59.

35. Seeman M. Alienation and anomie. In: Measures of personality and social psychological attitudes. Vol. 1. Robinson JR, et al. (eds.). Academic Press, San Diego 1991; 291-372.

36. Thompson SC, et al. The other side of perceived control: disadvantages and negative effects. In: The Social Psychology of Health. Spacapan S, Oshkamp S (eds). Sage, Beverly Hills 1988; 69-93.

37. Evans GW, Shapiro D, Lewis MA, et al. Specifying dysfunctional mismatches between different control dimensions. Br J Psychol 1993; 84: 255-73.

38. Degood DE. Cognitive control factors in vascular stress responses. Psychophysiology 1975; 12: 399-401.

39. Houston BK. Control over stress, locus of control, and response to stress. J Pers Soc Psychol 1972; 21: 249-55.

40. Manuck SB, Harvey AH, Lechleiter SL, Neal SK. Effects of coping on blood pressure responses to threat of aversive stimulation. Psychophysiology 1978; 15: 544-9.

41. LeBrón AM, Schulz AJ, Mentz G, White Perkins D. John Henryism, socioeconomic position, and blood pressure in a multi-ethnic urban community. Ethn Dis 2015; 25: 24-30. 